\title{
Modeling of the meteoroid stream of comet C/1975 T2 and $\lambda$-Ursae Majorids
}

\author{
M. Hajduková ${ }^{1}$ and L. Neslušan ${ }^{2}$ \\ ${ }^{1}$ Astronomical Institute, Slovak Academy of Science, Dúbravská cesta 9, 84504 Bratislava, Slovakia \\ e-mail: astromia@savba.sk \\ 2 Astronomical Institute, Slovak Academy of Science, 05960 Tatranská Lomnica, Slovakia \\ e-mail: ne@ta3.sk
}

Received 5 April 2019 / Accepted 14 May 2019

\begin{abstract}
Aims. We study the meteoroid stream of the long-period comet C/1975 T2 (Suzuki-Saigusa-Mori). This comet was suggested as the parent body of the established $\lambda$-Ursae Majorid meteor shower, No. 524.

Methods. We modeled 32 parts of a theoretical meteoroid stream of the parent comet considered. Each of our models is characterized with a single value of the evolutionary time and a single value of the strength of Poynting-Robertson effect. The evolutionary time ranges from 10000 to $80000 \mathrm{yr}$. It is the period during which the evolution of the stream part is followed. In each model, the dynamical evolution of 10000 test particles was then followed, via a numerical integration, from the time of the modeling up to the present. At the end of the integration, we analyzed the mean orbital characteristics of particles in the orbits that approach the Earth's orbit, which thus enabled us to predict a shower related to the parent comet. The predicted shower was subsequently compared with its observed counterparts. We separated the latter from the databases of real meteors. As well, we attempted to identify the predicted shower to a shower recorded in the International Astronomical Union Meteor Data Center (IAU MDC) list of all showers.

Results. Almost all modeled parts of the stream of comet C/1975 T2 are identified with the corresponding real shower in three videometeor databases. No real counterpart is found in the IAU MDC photographic or radio-meteor data. In the IAU MDC list of showers and in our current study, this shower is identified with the established $\lambda$-Ursae Majorid shower, No. 524. Hence, our modeling confirms the results of previous authors. At the same time we exclude an existence of other meteor shower associated with C/1975 T2.
\end{abstract}

Key words. comets: individual: C/1975 T2 - meteorites, meteors, meteoroids

\section{Introduction}

Several periodic comets are known to be the parent bodies of meteoroid streams, since we detect the corresponding meteor showers in the Earth's atmosphere. Probably, a prevailing part of periodic comets are the source of meteoroid material. However, a lot of comets have not been studied in the context of their potential meteoroid stream. In our work, which started about two decades ago (Neslušan 1999), we aim to increase the number of examined comets in this context

In this paper, we assume that the nucleus of long-period comet C/1975 T2 (Suzuki-Saigusa-Mori) could be a source of meteoroid particles. Subsequently, we model the meteoroid stream of the comet and follow its dynamical evolution in course to reveal a real meteor shower related to this comet.

Our paper is similar to recent similar studies of the relationship between various meteoroid streams and their parent bodies, i.e., cometary (Hajdukova et al. 2015; Ishiguro et al. 2015; Kornoš et al. 2015; Rudawska et al. 2016; Abedin et al. 2015, 2017, 2018; Babadzhanov et al. 2017; Jenniskens et al. 2017; Šegon et al. 2017) and lately also asteroidal (Babadzhanov et al. 2015a,b; Jopek 2015; Jopek \& Williams 2015; Rudawska \& Vaubaillon 2015; Olech et al. 2016; Wiegert et al. 2017; Dumitru et al. 2018; Sergienko et al. 2018a,b; Ye 2018; Guennoun et al. 2019; Ryabova et al. 2019). Some authors have attempted to work out or improve a method of prediction of particular shower, often on the basis of known parent body (Koten \& Vaubaillon 2015; Ryabova 2016; Sugar et al. 2017; Vaubaillon 2017;
Ryabova \& Rendtel 2018a,b). All this effort is highly desirable in the current era when a number of new showers as well as a number of new members of known showers are reported every year (e.g., Jones 2018; Jenniskens et al. 2018; Koukal 2018; Molau et al. 2018a,b,c; Shiba et al. 2018; Toth et al. 2018; Vida et al. 2018a,b; Wisniewski et al. 2018, if we consider only the last year).

Comet C/1975 T2 was observed within 90 days in October 1975. Immediately after the discovery of the comet, V. Guth and I. Hasegawa suggested the possibility of observing meteors associated with the comet passing its descending node on October, 31.4 UT, from the radiant RA $=158 \mathrm{deg}$, Dec $=+47 \mathrm{deg}$ and with $V_{\text {inf }}=62 \mathrm{~km} \mathrm{~s}^{-1}$ (Muirden et al. 1975). However, there were no records of any observation of such a shower in the literature until recently. In 2013, a new meteor shower with similar parameters was announced by Andreić et al. (2013), searching the Croatian Meteor Network and the SonotaCo databases. The shower was named $\lambda$-Ursae Majorids and received Number 524 in the International Astronomical Union Meteor Data Center (IAU MDC) list of showers ${ }^{1}$ (Jopek \& Kaňuchová 2014). The shower was confirmed by Jenniskens et al. (2016a). Andreić et al. (2013) also searched for a possible parent body of their newly found shower (No. 524) and proposed the comet C/1975 T2. This comet was also suggested as a parent body of another meteor shower No. 333, October Ursae Majorids, by Gajdoš (2008).

\footnotetext{
1 http://www.ta3.sk/IAUC22DB/MDC2007/Roje/roje_lista. php? corobic_roje=1\&sort_roje $=0$
} 

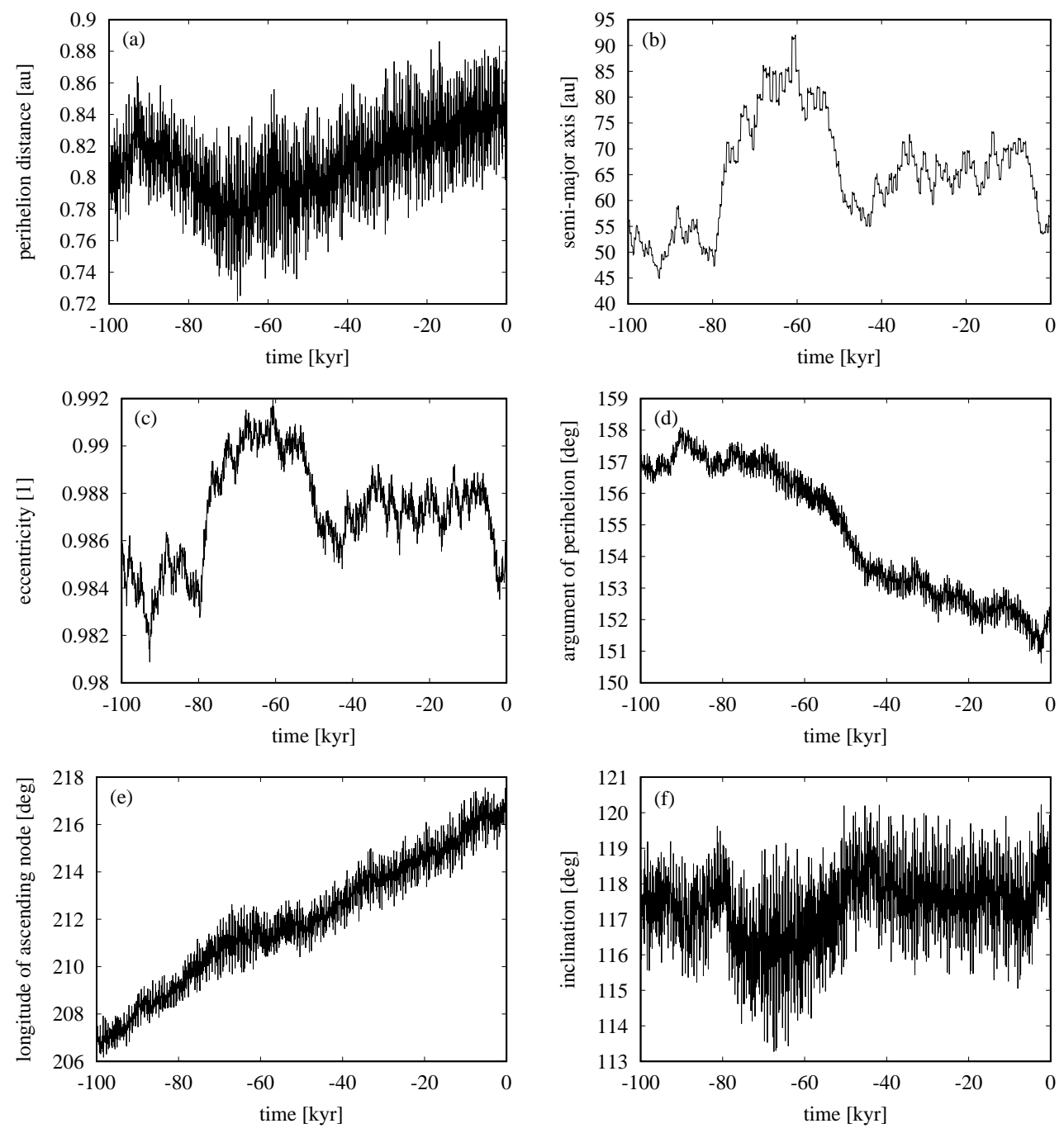

Fig. 1. Behavior of perihelion distance $(a)$, semimajor axis $(b)$, eccentricity $(c)$, argument of perihelion $(d)$, longitude of ascending node $(e)$, and inclination to the ecliptic $(f)$ of the initially nominal orbit of comet C/1975 T2 (Suzuki-Saigusa-Mori). The evolution is reconstructed backward for $100000 \mathrm{yr}$. A nongravitational force is ignored.

\section{Nominal orbit of comet $\mathrm{C} / 1975 \mathrm{~T} 2$}

In our study, we consider the orbit of comet C/1975 T2 with the orbital elements published in the JPL small-body browser (Giorgini et al. 1996) ${ }^{2}$. This orbit, referred to epoch 2014 November 14.0 (JDT $=2456975.5)$, has elements: $q=$ $0.838047 \mathrm{au}, e=0.985653, a=58.4126995 \mathrm{au}, \omega=152.0192^{\circ}$, $\Omega=216.8053^{\circ}, i=118.2332^{\circ}$, and $T=2442700.8602(1975$ October 15.3602). An uncertainty of the orbit determination is unknown. Hereinafter, this orbit is referred to as the nominal orbit. The orbital period of the comet in the nominal orbit is $446.00 \mathrm{yr}$ and its aphelion is situated in the distance of 115.9873520 au from the Sun.

The variation of the orbital elements of C/1975 T2 during the last $100 \mathrm{kyr}$ is shown in Fig. 1. In this period, only minor changes of perihelion distance (Fig. 1a), eccentricity (Fig. 1b), and inclination (Fig. 1f) have happened. In Figs. 1d and e, we can observe a decrease (increase) about several degrees in argument of perihelion (longitude of ascending node), respectively. Only the semimajor axis (Fig. 1b) has changed largely. Hence, it is also possible to expect a large change of these orbital elements at the particles of the meteoroid stream of the comet.

2 http://ssd.jpl.nasa.gov/sbdb.cgi

A73, page 2 of 8
The variation of the elements, although not very large, causes a significant change of the position of the ascending node of the orbit as seen in Fig. 2. The position of the descending node is almost the same and this node is situated closely at the Earth's orbit (green circle in Fig. 2). This circumstance favors an occurrence of a meteor shower.

The minimum distance between the orbits of the comet and Earth is also investigated. The behavior of this distance is shown in Fig. 3 for the period of the last $100 \mathrm{kyr}$. While the postperihelion arc is situated in a large heliocentric distance, close to the orbit of Saturn, the pre-perihelion arc is near the Earth's orbit $(\approx 0.1 \mathrm{au})$ during the whole investigated period. It implies an existence of only a single meteor shower on Earth.

\section{Stream modeling}

\subsection{Brief description of theory}

The theoretical stream of C/1975 T2 is modeled in the way that was firstly used by Neslušan (1999). The procedure was later slightly modified by Tomko \& Neslušan (2012). A detailed description was recently again given in Tomko \& Neslušan (2019). Therefore, we only briefly describe its main steps. At first, the orbit of the parent body is integrated in time backward 


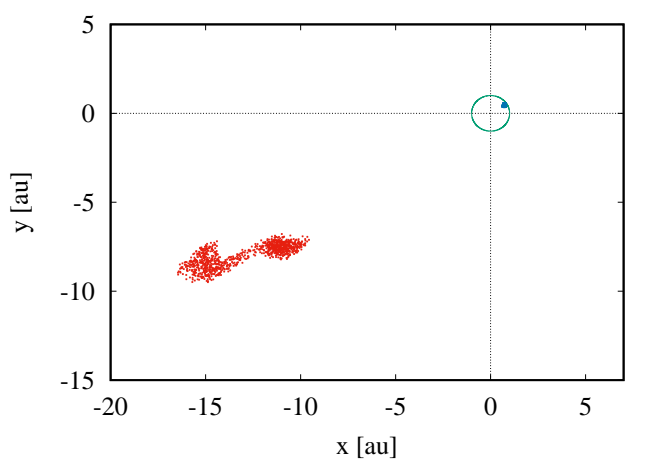

Fig. 2. Positions of ascending (red points) and descending (blue points) nodes of the orbit of comet C/1975 T2 during the last $100000 \mathrm{yr}$. The green circle indicates the orbit of the Earth.

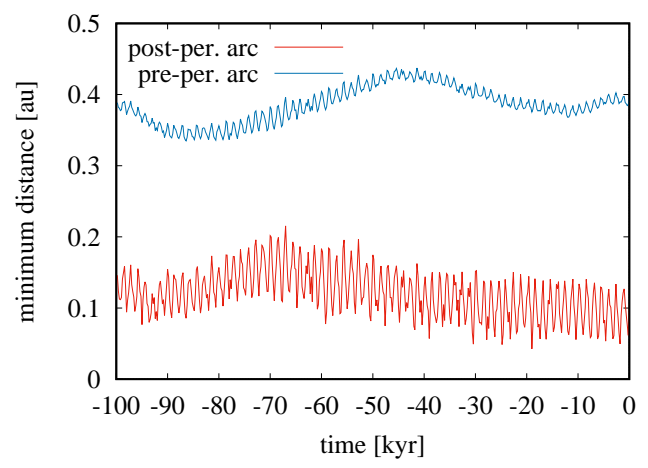

Fig. 3. Evolution of the minimum distance between the orbital arcs of comet C/1975 T2 and Earth's orbit from time $100000 \mathrm{yr}$ before the present to the present. The minimum distance of post-perihelion (pre-perihelion) arc is shown by the red (blue) curve.

to the moment of the perihelion passage of the body, which happened closest to an arbitrarily chosen time $t_{\mathrm{ev}}$. At perihelion, a cloud of 10000 test particles around the parent body is modeled and all this assembly is integrated in time forward, up to the present. At the end of the integration, the particles that move in the orbits that approach the Earth's orbit within $0.05 \mathrm{au}$ are selected. The characteristics of these particles correspond to those of expected shower and, thus, they enable us to predict the mean characteristics of the expected shower.

In our modeling, the numerical integration was performed via the integrator RA15 (Everhart 1985) within the software package MERCURY ${ }^{3}$ (Chambers 1999; Chambers \& Murison 2000). We took the gravitational perturbations of eight planets, Mercury to Neptune, into account.

In the models, the dynamics of the particles is assumed to be influenced by the Poynting-Robertson ( $\mathrm{P}-\mathrm{R}$, hereinafter) effect. The term $\mathrm{P}-\mathrm{R}$ effect is used to refer to the action of radial electromagnetic radiation pressure and the velocity-dependent effects on the meteoroid particles. Specifically, we used the formulas published by Klačka (2014).

The strength of the $\mathrm{P}-\mathrm{R}$ effect is given by parameter $\beta$, which is the ratio of the accelerations due to both the P-R effect and the gravity by the Sun. This parameter depends on the properties of particles, such as the size (geometrical cross section), density, light scattering efficiency (then on albedo and light absorption ability), and mass. In the case of meteoroids, these properties are rather uncertain. So, we rather regard $\beta$ as a free parameter and search, as far as possible, for its value to achieve the

3 https://ascl.net/1201.008 best agreement between the predicted and observed characteristics of found filaments of the investigated stream related to the considered parent body.

We created a series of models for various combinations of specific values of evolutionary time $t_{\mathrm{ev}}$ and parameter $\beta$. In reality, parameter $\beta$ ranges in a wider interval of values since the stream consists of the particles of various sizes and densities. As well, the particles are released in various times, therefore their evolutionary time must be different and can also acquire the values from a wider interval. Hence, the particular model we created does not represent a whole stream. The model of a whole stream is a composition of partial models, which give a good prediction of the corresponding real showers.

In more detail, we created the models for all combinations of values $t_{\mathrm{ev}}=10,20,40$, and $80 \mathrm{kyr}$ and $\beta=10^{-11}$ (this value implies no P-R effect, in fact), 0.0001, 0.001, 0.003, $0.005,0.007$, and 0.009 . We also calculated the models characterized with $\left(t_{\mathrm{ev}}, \beta\right)$ equal to $(10 \mathrm{kyr}, 0.006),(20 \mathrm{kyr}, 0.008)$, (40 kyr, 0.008), and ( $80 \mathrm{kyr}, 0.008)$ to find a critical upper value of $\beta$, for given $t_{\mathrm{ev}}$, for which the stream is completely deflected from the vicinity of the Earth's orbit and no shower is predicted.

\subsection{Results of modeling}

It appears that the stream of C/1975 T2 crosses the orbit of our planet only in its single section, therefore only a single observable meteor shower, originating from this comet, is predicted. The mean geophysical characteristics of this shower are given in Table 1 and the orbital elements of its mean orbit in Table 2.

We note that the shower is predicted to be rather compact and has a high geocentric velocity that is $62-63 \mathrm{~km} \mathrm{~s}^{-1}$. Its radiant area is elongated, in time of corresponding to the mean solar longitude, about $80^{\circ}$ from the Sun. Hence, it can also be observed on the night sky, although it is not a typical night-time shower. The mean solar longitude of the shower as predicted by individual models ranges from $208^{\circ}$ to $215^{\circ}$ (this corresponds to October 21 to 28 ).

The shower was predicted in the model with every considered evolutionary time, $t_{\mathrm{ev}}$, from 10 to $80 \mathrm{kyr}$. For each considered evolutionary time, the large particles, not detectably influenced by the P-R effect, could come to the vicinity of the Earth's orbit and, thus, collide with this planet. In Fig. 4a, we can see that there is practically no shift of the radiant area with the increasing evolutionary time. The size of the area is, however, increasing with increasing $t_{\mathrm{ev}}$.

The upper value of $\mathrm{P}-\mathrm{R}$-effect strength, $\beta$, is found to be $0.005,0.007,0.008$, and 0.008 in models for $t_{\mathrm{ev}} 10,20,40$, and $80 \mathrm{kyr}$, respectively. As seen in Fig. $4 \mathrm{~b}$ in the case of models for $t_{\mathrm{ev}}=20 \mathrm{kyr}$, the radiants are slightly moved to lower values of right ascension by the P-R effect. A similar trend is found for the other evolutionary times.

\section{Identification of the predicted shower with its real counterpart}

The dynamical characteristics of the predicted shower were used to start a search for the corresponding real shower. We searched the real shower (1) in the databases of meteor orbits and (2) in the IAU MDC list of all showers.

\subsection{Separation of real shower from databases}

We searched for a corresponding shower in the following meteor databases: (1) the IAU MDC photographic database, 
Table 1. Geophysical parameters of the parts of predicted shower.

\begin{tabular}{|c|c|c|c|c|c|c|c|c|c|c|}
\hline $\begin{array}{c}t_{\mathrm{ev}} \\
(\mathrm{kyr})\end{array}$ & $\beta$ & $\begin{array}{l}\left\langle\lambda_{\odot}\right\rangle \\
(\operatorname{deg})\end{array}$ & $\begin{array}{c}\lambda_{\odot, \min }-\lambda_{\odot, \max } \\
(\mathrm{deg})\end{array}$ & $\begin{array}{c}\langle\alpha\rangle \\
(\mathrm{deg})\end{array}$ & $\begin{array}{c}\langle\delta\rangle \\
(\mathrm{deg})\end{array}$ & $\begin{array}{c}\left\langle V_{\mathrm{g}}\right\rangle \\
\left(\mathrm{km} \mathrm{s}^{-1}\right)\end{array}$ & $\begin{array}{r}\left\langle V_{\mathrm{h}}\right\rangle \\
\left(\mathrm{km} \mathrm{s}^{-1}\right)\end{array}$ & $\begin{array}{r}\langle\gamma\rangle \\
(\operatorname{deg})\end{array}$ & $n$ & $D_{1}$ \\
\hline 10 & & $4.1+0.7$ & $211.8-216.6$ & $53.7 \pm 1.3$ & $7.8 \pm 0.3$ & $62.6 \pm 0.3$ & $42.2 \pm 0.0$ & 80 & 2233 & \\
\hline 10 & & & & & & & & & & \\
\hline 10 & & & & & & & & 80 & 3675 & \\
\hline 10 & & & & & & & 0 & 80 & 3762 & \\
\hline 10 & & & & & & & \pm & 80 & 820 & \\
\hline 20 & & & & & & & $1 \pm$ & 79 & 6031 & \\
\hline 20 & 0.0001 & & & & 0.6 & & ) & 79 & 06 & \\
\hline 20 & & & & & & & 2 & 79 & 834 & \\
\hline 20 & & & & & & & & 79 & 462 & \\
\hline 20 & & & & & & & \pm & 79 & 2792 & \\
\hline 20 & & & & & & & & 79 & 149 & \\
\hline 40 & $10^{-11}$ & & & & & & $1 \pm$ & 80 & 4330 & \\
\hline 40 & & & & & & & & 80 & 4440 & \\
\hline 40 & & & & & & & ) & 79 & 5272 & \\
\hline 40 & 0 . & & & & & & $2 \pm$ & 79 & 5027 & \\
\hline 40 & & & & & & & & 80 & 3260 & \\
\hline 40 & & & & & & & & & 383 & \\
\hline 40 & & & & & & & $2 \pm$ & & 14 & \\
\hline 80 & $10^{-11}$ & & & & .0 & 62.4 & $1+$ & 80 & 2380 & \\
\hline 80 & 0.000 & & & & $47.1 \pm 2.3$ & & $2.1 \pm 0.1$ & 79 & 2583 & \\
\hline 80 & 0.001 & $214.2 \pm 3.8$ & & & $47.8 \pm 1.8$ & $62.4 \pm 0.6$ & $42.1 \pm 0.1$ & 79 & 2978 & \\
\hline 80 & & & & & & & & & & \\
\hline 80 & & & & & & & & & 2723 & \\
\hline 80 & & & & $146.2 \pm 1.9$ & & & $42.2 \pm 0.0$ & 80 & 563 & \\
\hline 80 & 0.008 & $207.8 \pm 0.8$ & $205.2-231.8$ & $144.8 \pm 1.3$ & $49.8 \pm 0.4$ & $63.1 \pm 0.3$ & $42.2 \pm 0.0$ & 80 & 22 & \\
\hline
\end{tabular}

Notes. The following denotation is used: $t_{\mathrm{ev}}-$ the evolutionary time (time before the present when the part of the stream was modeled); $\beta$ - the parameter characterizing the strength of P-R effect; $\left\langle\lambda_{\odot}\right\rangle$ - mean solar longitude; $\lambda_{\odot \text {,min }}$ and $\lambda_{\odot \text {,max }}$ - period of shower activity in term of minimum and maximum solar longitude of shower occurrence; $\langle\alpha\rangle$ and $\langle\delta\rangle-$ mean equatorial coordinates of geocentric radiant; $\left\langle V_{\mathrm{g}}\right\rangle$ and $\left\langle V_{\mathrm{h}}\right\rangle-$ mean geocentric and heliocentric velocities; $\langle\gamma\rangle$ - angular distance of mean radiant from the Sun in time corresponding to $\left\langle\lambda_{\odot}\right\rangle ; n-$ number of particles in the predicted shower; and $D_{\mathrm{lim}}$ - the limiting value of the Southworth-Hawkins $D_{\mathrm{SH}}$-discriminant to select the shower.

version-2013 (Porubčan et al. 2011; Neslušan et al. 2014), (2) the IAU MDC Cameras for Allsky Meteor Surveillance (CAMS) video database (Gural 2011; Jenniskens et al. 2011, 2016b,a,c; Jenniskens \& Nénon 2016), (3) the 2007-2015 SonotaCo video (SonotaCo 2009, 2016), the EDMOND video (Kornoš et al. 2014a,b), and (5) the radio-meteor database (Hawkins 1963; Sekanina \& Southworth 1975; Lindblad 2003, priv. comm.). These databases contain 4873, $110521,208826,145830$, and 62907 records on meteor orbits and geophysical data, respectively. We briefly refer to these databases with the capital letters F, C, S, E, and R, respectively.

To select the meteors of searched shower from the given database, we used the "break-point method" (Neslušan et al. 1995, 2013). We briefly describe the main steps of the method, which is based on an analysis of the dependence of the number of selected shower meteors on the limiting value of the SouthworthHawkins (Southworth \& Hawkins 1963) $D_{\mathrm{SH}}$ discriminant, $D_{\mathrm{lim}}$.

In the first step, we calculate the values $D_{\mathrm{SH}}$ between the mean orbit of predicted shower and every meteor in the database considered. If $D_{\mathrm{SH}}$ is smaller than a chosen limiting value, $D_{\mathrm{lim}}$, then the meteor is regarded as the member of the shower. Then, we calculate the mean orbit of the separated group of shower meteors and, in the second step, $D_{\mathrm{SH}}$ between this new mean orbit and orbit of every meteor in the database is calculated. And, considering the meteors separated in this step, we again calculate their mean orbit, which is again the reference orbit for the new selection, etc. We terminate this iteration procedure, when the difference between two successive mean orbits is negligible. In the final separation, we obtain $N$ shower meteors.

The above sketched iteration procedure is performed for a series of limiting values $D_{\text {lim }}$, usually $0.01,0.02,0.03, \ldots, 0.50$. Having the corresponding numbers of separated meteors, we can construct the dependence $N=N\left(D_{\text {lim }}\right)$. If the corresponding real shower was recorded in the database, the dependence exhibits a convex behavior followed by a constant or only moderately increasing behavior - a plateau. The value of $D_{\text {lim }}$ corresponding to the beginning of the plateau is the most appropriate to separate the densest core of the shower from the database. In other words, the actual members of the shower in our search are the meteors separated within the iteration procedure using this critical $D_{\text {lim. }}$.

In reality, a separated shower can be low-numerous and dispersed and the plateau can be short and hardly discernible. Unfortunately, this also occurred in the case of the real shower related to $\mathrm{C} / 1975 \mathrm{~T} 2$. We show the $N=N\left(D_{\text {lim }}\right)$-dependence obtained in course to separate a real shower from the CAMS database in Fig. 5. This shower corresponds to the prediction by model with $t_{\mathrm{ev}}=40 \mathrm{kyr}$ and $\beta=0.003$. A small plateau begins at $D_{\text {lim }}=0.13$. It is pointed out by an arrow. Using value $D_{\lim }=0.13$, a relatively small number, 19 , of the shower 
Table 2. Mean orbital elements of the parts of predicted shower.

\begin{tabular}{|c|c|c|c|c|c|c|c|}
\hline $\begin{array}{c}t_{\mathrm{ev}} \\
(\mathrm{kyr})\end{array}$ & $\beta$ & $\begin{array}{l}\langle q\rangle \\
(\mathrm{au})\end{array}$ & $\begin{array}{l}\langle a\rangle \\
(\mathrm{au})\end{array}$ & $\langle e\rangle$ & $\begin{array}{c}\langle\omega\rangle \\
(\mathrm{deg})\end{array}$ & $\begin{array}{c}\langle\Omega\rangle \\
(\operatorname{deg})\end{array}$ & $\begin{array}{c}\langle i\rangle \\
(\operatorname{deg})\end{array}$ \\
\hline 10 & $10^{-11}$ & $0.892 \pm 0.014$ & $124.6 \pm 40.3$ & $0.992 \pm 0.003$ & $150.9 \pm 0.4$ & $214.4 \pm 0.8$ & $120.4 \pm 1.0$ \\
\hline 10 & 0.0001 & $0.893 \pm 0.015$ & $127.8 \pm 40.2$ & $0.992 \pm 0.003$ & $150.9 \pm 0.4$ & $214.3 \pm 0.8$ & $120.5 \pm 1.0$ \\
\hline 10 & 0.001 & $0.904 \pm 0.023$ & $158.3 \pm 52.3$ & $0.994 \pm 0.002$ & $150.7 \pm 0.4$ & $213.8 \pm 0.9$ & $121.2 \pm 1.2$ \\
\hline 10 & 0.003 & $0.912 \pm 0.025$ & $199.7 \pm 89.0$ & $0.995 \pm 0.002$ & $150.5 \pm 0.4$ & $213.4 \pm 0.9$ & $121.8 \pm 1.2$ \\
\hline 10 & 0.005 & $0.930 \pm 0.026$ & $315.2 \pm 118.5$ & $0.997 \pm 0.001$ & $150.3 \pm 0.5$ & $212.6 \pm 1.0$ & $122.7 \pm 1.2$ \\
\hline 20 & $10^{-11}$ & $0.901 \pm 0.019$ & $79.7 \pm 30.6$ & $0.987 \pm 0.004$ & $149.5 \pm 0.9$ & $215.2 \pm 1.1$ & $119.1 \pm 1.2$ \\
\hline 20 & 0.0001 & $0.901 \pm 0.019$ & $80.2 \pm 30.7$ & $0.987 \pm 0.004$ & $149.5 \pm 0.9$ & $215.1 \pm 1.1$ & $119.2 \pm 1.3$ \\
\hline 20 & 0.001 & $0.904 \pm 0.020$ & $97.4 \pm 38.4$ & $0.989 \pm 0.004$ & $149.4 \pm 0.8$ & $214.5 \pm 1.2$ & $119.6 \pm 1.3$ \\
\hline 20 & 0.003 & $0.913 \pm 0.024$ & $143.2 \pm 66.6$ & $0.992 \pm 0.003$ & $149.3 \pm 0.7$ & $213.4 \pm 1.1$ & $120.4 \pm 1.3$ \\
\hline 20 & 0.005 & $0.922 \pm 0.024$ & $201.9 \pm 132.3$ & $0.995 \pm 0.002$ & $149.3 \pm 0.6$ & $212.7 \pm 0.9$ & $121.0 \pm 1.3$ \\
\hline 20 & 0.007 & $0.925 \pm 0.024$ & $557.2 \pm 638.9$ & $0.998 \pm 0.001$ & $149.6 \pm 0.5$ & $212.2 \pm 1.1$ & $121.1 \pm 1.2$ \\
\hline 40 & $10^{-11}$ & $0.904 \pm 0.020$ & $80.7 \pm 40.2$ & $0.986 \pm 0.006$ & $150.3 \pm 1.4$ & $215.4 \pm 2.0$ & $119.5 \pm 1.4$ \\
\hline 40 & 0.0001 & $0.904 \pm 0.021$ & $83.0 \pm 42.1$ & $0.987 \pm 0.006$ & $150.3 \pm 1.4$ & $215.3 \pm 2.1$ & $119.5 \pm 1.4$ \\
\hline 40 & 0.001 & $0.906 \pm 0.021$ & $98.5 \pm 49.1$ & $0.989 \pm 0.005$ & $150.1 \pm 1.3$ & $214.3 \pm 1.8$ & $119.7 \pm 1.3$ \\
\hline 40 & 0.003 & $0.914 \pm 0.025$ & $141.7 \pm 80.3$ & $0.992 \pm 0.004$ & $150.0 \pm 1.2$ & $212.7 \pm 1.5$ & $120.4 \pm 1.4$ \\
\hline 40 & 0.005 & $0.921 \pm 0.025$ & $189.8 \pm 166.4$ & $0.994 \pm 0.003$ & $150.1 \pm 0.9$ & $211.7 \pm 1.1$ & $121.0 \pm 1.2$ \\
\hline 40 & 0.007 & $0.931 \pm 0.025$ & $358.5 \pm 226.2$ & $0.997 \pm 0.002$ & $150.7 \pm 0.7$ & $210.9 \pm 0.9$ & $121.3 \pm 1.3$ \\
\hline 40 & 0.008 & $0.932 \pm 0.026$ & $975.1 \pm 264.7$ & $0.999 \pm 0.000$ & $151.3 \pm 0.4$ & $210.9 \pm 1.0$ & $121.1 \pm 1.2$ \\
\hline 80 & $10^{-11}$ & $0.906 \pm 0.023$ & $102.3 \pm 78.6$ & $0.987 \pm 0.007$ & $150.1 \pm 2.1$ & $216.1 \pm 4.1$ & $119.9 \pm 2.0$ \\
\hline 80 & 0.0001 & $0.906 \pm 0.023$ & $99.5 \pm 73.2$ & $0.987 \pm 0.007$ & $149.9 \pm 2.2$ & $216.3 \pm 4.5$ & $119.9 \pm 2.1$ \\
\hline 80 & 0.001 & $0.906 \pm 0.024$ & $119.4 \pm 83.2$ & $0.989 \pm 0.006$ & $149.5 \pm 2.2$ & $214.3 \pm 3.8$ & $119.9 \pm 1.9$ \\
\hline 80 & 0.003 & $0.910 \pm 0.026$ & $164.7 \pm 109.3$ & $0.992 \pm 0.005$ & $149.8 \pm 2.2$ & $211.3 \pm 2.7$ & $120.5 \pm 1.7$ \\
\hline 80 & 0.005 & $0.917 \pm 0.028$ & $212.1 \pm 207.5$ & $0.994 \pm 0.003$ & $150.3 \pm 1.9$ & $209.7 \pm 1.8$ & $121.0 \pm 1.5$ \\
\hline 80 & 0.007 & $0.927 \pm 0.027$ & $315.2 \pm 290.3$ & $0.996 \pm 0.002$ & $151.3 \pm 1.1$ & $208.5 \pm 1.1$ & $121.6 \pm 1.3$ \\
\hline 80 & 0.008 & $0.943 \pm 0.021$ & $677.0 \pm 425.7$ & $0.998 \pm 0.001$ & $152.5 \pm 0.4$ & $207.8 \pm 0.9$ & $121.8 \pm 1.0$ \\
\hline
\end{tabular}

Notes. The parameter $t_{\mathrm{ev}}$ is the evolutionary time (time before the present when the part of the stream was modeled) and $\beta$ is the parameter characterizing the strength of $\mathrm{P}-\mathrm{R}$ effect.

meteors were separated. A larger number of shower meteors could be separated by using $D_{\text {lim }}=0.20$, at which the curve of $N=N\left(D_{\text {lim }}\right)$-dependence is again, a little, broken. This second modification of obviously the same shower consists of 26 meteors.

A similar situation could be observed at the separations from the SonotaCo and EDMOND video databases. In total, we separated six modifications of the single real shower from these data sets, that is, one less and one more numerous modification from each data set. The mean geophysical parameters of these modifications are given in Table 3 and mean orbits in Table 4. The positions of radiants of meteors in all modifications are shown in Fig. 4c.

We attempted to separate the real shower from the IAU MDC photographic and radio-meteor databases as well. In the photographic data, three or less meteors were separated for $D_{\text {lim }}<0.49$ (or for $D_{\text {lim }}<0.44$ in few models with a large $\beta$ ). This number is too small to regard the group of separated meteors as a shower. And, a more numerous shower (consisting typically of 13 meteors), separated for the unacceptably high value of $D_{\text {lim }}=0.49$ (also $D_{\text {lim }}=0.44$ ), was too different to be related to its predicted counterpart. The value of $D_{\text {lim }}$ should not exceed $\sim 0.25$ for a well-defined shower.

A similar $N=N\left(D_{\text {lim }}\right)$-dependence was observed in all separations from the radio-meteor data. The number of separated shower meteors did not exceed three up to $D_{\lim }=0.34$. The shower separated at the high value of $D_{\text {lim }}=0.35$, again, was unlike to any predicted shower. We did not regard it as related to C/1975 T2.

The iteration procedure might sometimes significantly change the mean orbit of the separated shower from its prediction. After the separation, it is therefore necessary to evaluate a measure of relationship between the predicted, theoretical, and separated real shower. We did this evaluation suggesting and performing the following empirical procedure. We calculated the mean value of the Southworth-Hawkins D-discriminant, $\left\langle D_{\mathrm{mj}}\right\rangle$, between the mean orbit of real shower and each orbit of its members and its statistical uncertainty, $\sigma_{\mathrm{D}}$. We suppose that the orbit of any member of the predicted shower should not differ from the orbit of at least one meteor of real shower more than about $\left\langle D_{\mathrm{mj}}\right\rangle+\sigma_{\mathrm{D}}$, when the diversity is evaluated with the help of the $D_{\mathrm{SH}}$ criterion. We added $\sigma_{\mathrm{D}}$, since some meteors of the shower itself at the border of its orbital phase space would not differ within $\left\langle D_{\mathrm{mj}}\right\rangle$ alone. Subsequently, we calculated $D_{\mathrm{SH}}$ between every pair of the orbits of both theoretical particle and meteor of real shower. If $D_{\mathrm{SH}} \leq\left(\left\langle D_{\mathrm{mj}}\right\rangle+\sigma_{\mathrm{D}}\right)$ at least once, then the particle of predicted shower is regarded as matching the real shower. The values of $\left\langle D_{\mathrm{mj}}\right\rangle$ with their sigmas for the separated modifications of real showers are given in the last column of Table 3.

Let us denote the number of the matching particles as $n_{\text {yes }}$ and the number of the other particles as $n_{\text {no }}$, where the sum $n_{\text {yes }}+$ $n_{\text {no }}$ equals the total number of the particles of predicted shower. The measure of the agreement of corresponding theoretical and 

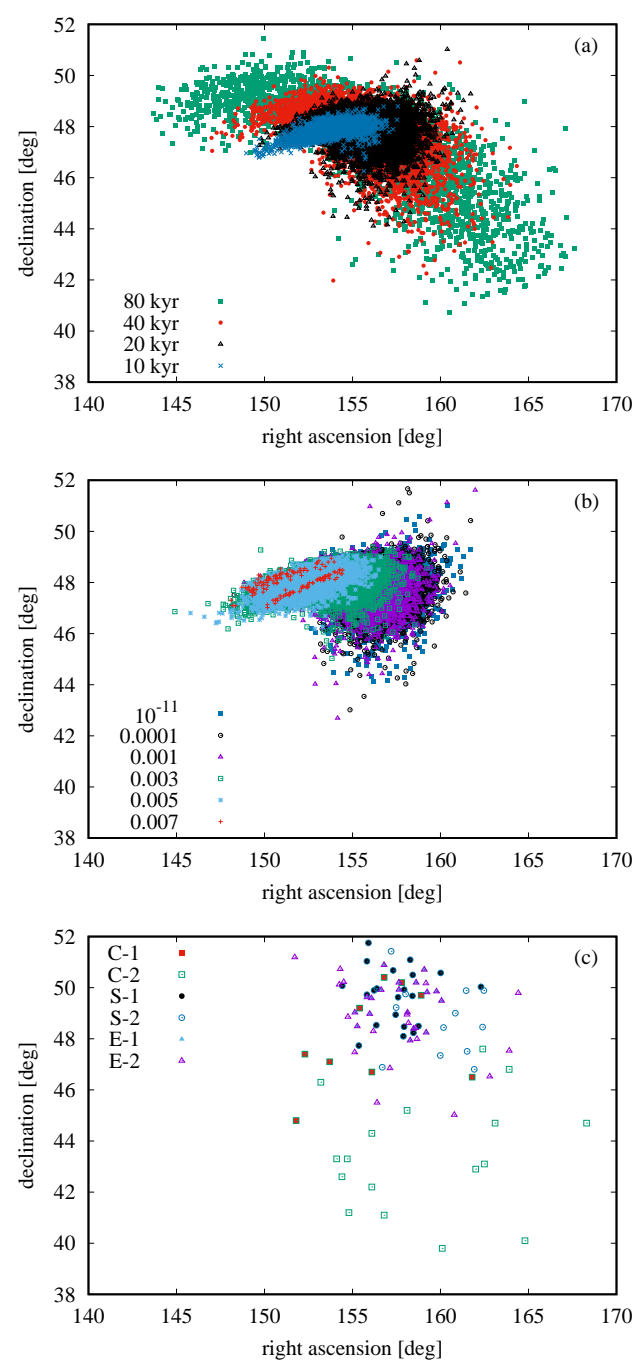

Fig. 4. Positions of predicted radiants in various models and radiants of real meteors. Panel a: positions in the models for the P-R-effect parameter $\beta=10^{-11}$ and a series of evolutionary times, 10, 20,40, and $80 \mathrm{kyr}$ are shown. Panel $b$ : positions in the models for the evolutionary time of $20 \mathrm{kyr}$ and series of parameters $\beta$ equal to $10^{-11}, 0.0001$, $0.001,0.003,0.005$, and 0.007 can be seen. Panel $c$ : radiant positions of the meteors constituting the real shower, related to comet $\mathrm{C} / 1975$ T2, which was separated in six modifications from three catalogs, are shown. A given kind of symbol shows the positions of radiants in one modification.

real showers is then defined as

$\eta=\frac{n_{\mathrm{yes}}}{n_{\mathrm{yes}}+n_{\mathrm{no}}}$.

The values of $\eta$ for the individual models are given in Table 5. This information in a graphical form is illustrated in Fig. 6. Likely, we can regard the agreement as insufficient if $\eta<0.5$. In Table 5, we can see that only the model for $t_{\mathrm{ev}}=$ $80 \mathrm{kyr}$ and $\beta=0.008$ does not match the real meteor shower, in any of its modifications, related to the stream of C/1975 T2. Generally, the match is worse for high $\beta$-values as well as a high evolutionary time.

\subsection{Identification of real shower in the IAU MDC list}

The predicted shower was further identified with its real counterpart in the IAU MDC list of all showers (Jopek \& Kaňuchová

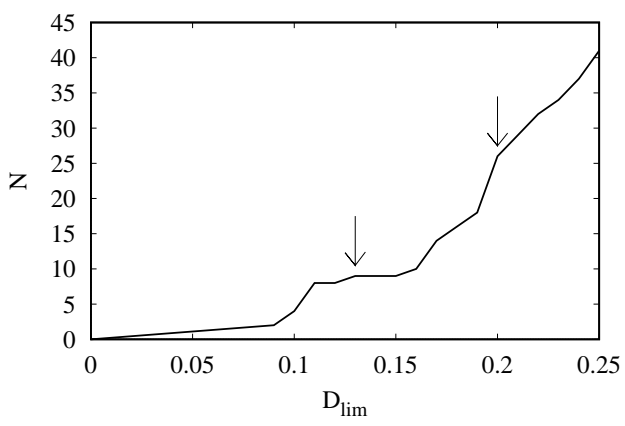

Fig. 5. Dependence of the number of real-shower meteors, $N$, on the limiting value of the Southworth-Hawkins $D$-discriminant, $D_{\lim }$. The meteors were separated from the CAMS video database. There seems to be two plateaus. Their beginnings (break points) are indicated with the arrows. The corresponding values of $D_{\text {lim }}$ were used to separate two modifications of the shower.
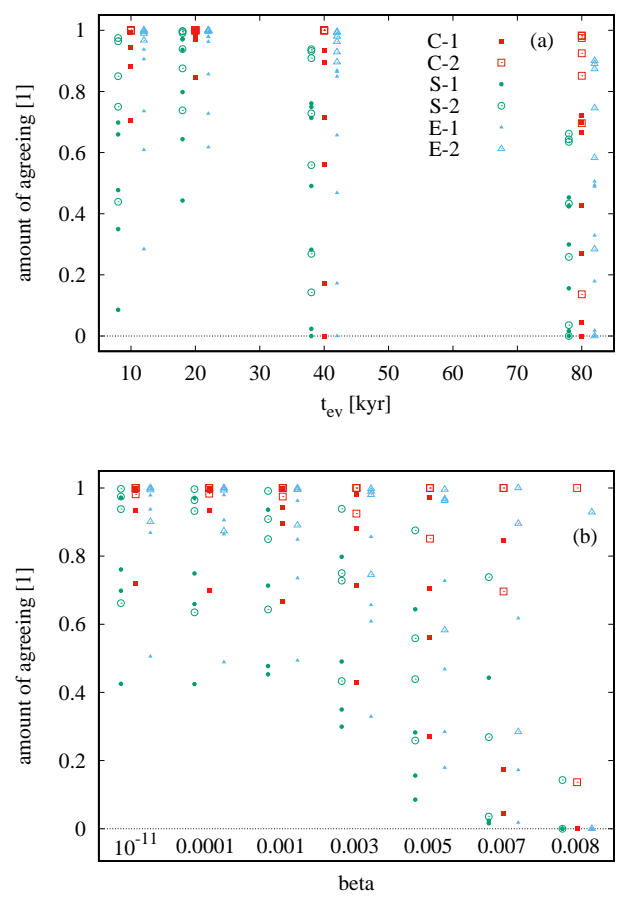

Fig. 6. Measure of agreement, $\eta$, between the predicted parts of shower related to C/1975 T2 and six modifications of the corresponding real shower. Each of the predicted parts is characterized with $t_{\mathrm{ev}}$ and $\beta$ (having the same meaning as in Tables 1 and 2). The modifications were separated from C, S, and E databases (see Tables 3 and 4). Panels a and $b$ : dependencies of $\eta$ on $t_{\mathrm{ev}}$ and $\beta$, respectively, are shown. For the sake of better transparency, the positions of symbols for $\mathrm{S}$ (E) catalog are artificially shifted left (right) relatively to their actual values. The symbols of given kind show the value of $\eta$ evaluating the agreement between the given modification of real shower (C-1, C-2, S-1, S-2, E-1, or E-2; see legend in the plot) and all predicted parts of shower (as listed in Tables 1 and 2).

2014). Since only the mean characteristics of the showers are available in the list, we identified the theoretical predictions, by the individual models, to the shower in the list using the $D_{\mathrm{SH}}$ discriminant. In more detail, we calculated the value of $D_{\mathrm{SH}}$ for every pair of orbits, where the first component of the pair was the mean orbit of predicted shower and the second component was the orbit in the list.

It appeared that the predicted shower, in all models, was identified to the established IAU MDC shower $\lambda$-Ursae 
Table 3. Mean geophysical parameters of the real meteor shower related to the stream of comet C/1975 T2.

\begin{tabular}{|c|c|c|c|c|c|c|c|c|c|}
\hline Mod. & $D_{\lim }$ & $\begin{array}{l}\left\langle\lambda_{\odot}\right\rangle \\
(\operatorname{deg})\end{array}$ & $\begin{array}{c}\lambda_{\odot, \min }-\lambda_{\odot, \max } \\
(\operatorname{deg})\end{array}$ & $\begin{array}{c}\langle\alpha\rangle \\
(\operatorname{deg})\end{array}$ & $\begin{array}{c}\langle\delta\rangle \\
(\operatorname{deg})\end{array}$ & $\begin{array}{c}\left\langle V_{\mathrm{g}}\right\rangle \\
\left(\mathrm{km} \mathrm{s}^{-1}\right)\end{array}$ & $\begin{array}{c}\left\langle V_{\mathrm{h}}\right\rangle \\
\left(\mathrm{km} \mathrm{s}^{-1}\right)\end{array}$ & $N$ & $\left\langle D_{\mathrm{mj}}\right\rangle$ \\
\hline $\mathrm{C}-1$ & 0.13 & $214.6 \pm 2.4$ & $211.5-220.5$ & $156.1 \pm 3.2$ & $48.0 \pm 1.9$ & $61.6 \pm 1.2$ & $41.8 \pm 0.6$ & 9 & $0.088 \pm 0.018$ \\
\hline $\mathrm{C}-2$ & 0.20 & $218.0 \pm 4.0$ & $211.5-225.3$ & $158.1 \pm 4.3$ & $45.0 \pm 3.0$ & $61.9 \pm 1.3$ & $41.2 \pm 0.8$ & 26 & $0.143 \pm 0.028$ \\
\hline S-1 & 0.08 & $214.4 \pm 1.1$ & $211.5-225.3$ & $157.5 \pm 1.7$ & $49.7 \pm 1.1$ & $60.8 \pm 0.7$ & $42.0 \pm 0.4$ & 21 & $0.057 \pm 0.016$ \\
\hline $\mathrm{S}-2$ & 0.11 & $215.0 \pm 1.6$ & $211.5-225.3$ & $158.4 \pm 2.2$ & $49.3 \pm 1.3$ & $60.7 \pm 0.8$ & $41.9 \pm 0.5$ & 33 & $0.071 \pm 0.021$ \\
\hline E-1 & 0.10 & $214.6 \pm 1.3$ & $211.4-225.3$ & $157.7 \pm 1.5$ & $49.4 \pm 0.8$ & $60.8 \pm 0.8$ & $41.9 \pm 0.4$ & 19 & $0.058 \pm 0.020$ \\
\hline E-2 & 0.15 & $215.0 \pm 2.3$ & $209.7-225.3$ & $157.7 \pm 2.7$ & $49.0 \pm 1.4$ & $60.5 \pm 0.9$ & $41.4 \pm 0.7$ & 36 & $0.092 \pm 0.034$ \\
\hline \multicolumn{2}{|c|}{ Andreić et al. (2013) } & 215.0 & & 157.2 & 49.4 & 60.3 & & 29 & \\
\hline \multicolumn{2}{|c|}{ Rudawska et al. (2015) } & $215.1 \pm 3.1$ & & $157.3 \pm 4.3$ & $49.0 \pm 2.4$ & $59.9 \pm 1.2$ & & 82 & \\
\hline \multicolumn{2}{|c|}{ Jenniskens et al. (2016a) } & 214.0 & & $157.8 \pm 1.1$ & $50.2 \pm 0.4$ & 60.9 & & 4 & \\
\hline \multicolumn{2}{|c|}{ Jenniskens et al. (2018) } & 214.6 & & 158.4 & 49.5 & 60.7 & & 33 & \\
\hline
\end{tabular}

Notes. Two modifications (for two threshold values of the Southworth-Hawkins D-discriminant, $D_{\text {lim }}$ ) of the shower were separated from each of three databases, C, S, and E. In addition, we also give the mean parameters of the showers in the IAU MDC list as published by various authors, those by Rudawska et al. (2015) included. Further symbols used as follows: $\left\langle\lambda_{\odot}\right\rangle$ - mean solar longitude; $\lambda_{\odot, \min }$ and $\lambda_{\odot, \text { max }}-$ period of shower activity in term of minimum and maximum solar longitude of shower occurrence; $\langle\alpha\rangle$ and $\langle\delta\rangle$ - mean equatorial coordinates of geocentric radiant; $\left\langle V_{\mathrm{g}}\right\rangle$ and $\left\langle V_{\mathrm{h}}\right\rangle$ - mean geocentric and heliocentric velocities; $N$ - number of meteors in given modification; and $\left\langle D_{\mathrm{mj}}\right\rangle-$ the mean value of the Southworth-Hawkins D-discriminant characterizing the dispersion of meteor orbits in given modification (see Sect. 4.1).

Table 4. Mean orbital elements of the real meteor shower related to the stream of comet C/1975 T2.

\begin{tabular}{lccccccc}
\hline \hline Mod. & $D_{\lim }$ & $\begin{array}{c}\langle q\rangle \\
(\mathrm{au})\end{array}$ & $\begin{array}{c}\langle a\rangle \\
(\mathrm{au})\end{array}$ & $\langle e\rangle$ & $\begin{array}{c}\langle\omega\rangle \\
(\mathrm{deg})\end{array}$ & $\begin{array}{c}\langle\Omega\rangle \\
(\mathrm{deg})\end{array}$ & $\begin{array}{c}\langle i\rangle \\
(\mathrm{deg})\end{array}$ \\
\hline $\mathrm{C}-1$ & 0.14 & $0.917 \pm 0.007$ & $245.9 \pm 428.4$ & $0.958 \pm 0.049$ & $147.5 \pm 1.6$ & $214.6 \pm 2.4$ & $118.3 \pm 3.9$ \\
$\mathrm{C}-2$ & 0.20 & $0.910 \pm 0.022$ & $91.8 \pm 268.0$ & $0.908 \pm 0.068$ & $145.9 \pm 4.8$ & $218.0 \pm 4.0$ & $121.4 \pm 4.9$ \\
S-1 & 0.08 & $0.920 \pm 0.007$ & $29.2 \pm 35.4$ & $0.976 \pm 0.035$ & $148.3 \pm 1.7$ & $214.4 \pm 1.1$ & $115.2 \pm 1.9$ \\
S-2 & 0.11 & $0.917 \pm 0.010$ & $22.2 \pm 30.9$ & $0.969 \pm 0.042$ & $147.6 \pm 2.3$ & $215.0 \pm 1.6$ & $115.1 \pm 2.0$ \\
E-1 & 0.11 & $0.918 \pm 0.009$ & $19.1 \pm 27.6$ & $0.966 \pm 0.036$ & $147.7 \pm 2.2$ & $214.6 \pm 1.3$ & $115.3 \pm 1.8$ \\
E-2 & 0.15 & $0.916 \pm 0.016$ & $15.0 \pm 21.0$ & $0.925 \pm 0.062$ & $146.9 \pm 3.5$ & $215.0 \pm 2.3$ & $115.7 \pm 2.2$ \\
\hline Andreić et al. (2013) & 0.917 & 13 & 0.931 & 147 & 215.0 & 115 \\
Rudawska et al. (2015) & $0.915 \pm 0.031$ & & $0.875 \pm 0.091$ & $147.1 \pm 7.3$ & $215.1 \pm 3.1$ & $115.8 \pm 2.7$ \\
Jenniskens et al. (2016a) & 0.920 & 3.10 & 1.008 & 148.5 & 213.5 & 114.5 \\
Jenniskens et al. (2018) & 0.915 & 41.3 & 0.978 & 147.1 & 214.6 & 114.7 \\
\hline
\end{tabular}

Notes. Two modifications (for two threshold values of the Southworth-Hawkins D-discriminant, $D_{\text {lim }}$ ) of the shower were separated from each of three databases, $\mathrm{C}, \mathrm{S}$, and E. In addition, we also give the mean elements of the orbits in the IAU MDC list as published by various authors, those by Rudawska et al. (2015) included.

Majorids, No. 524. In the IAU MDC list, version we used, this shower was given in three modifications (Andreić et al. 2013; Jenniskens et al. 2016a, 2018). The mean geophysical parameters of these modifications are given in Table 3 and mean orbits in Table 4.

At the identification, the value of $D_{\mathrm{SH}}$ varied from 0.089 to 0.195 (modification by Andreić et al. 2013), from 0.101 to 0.232 (modification by Jenniskens et al. 2016a), or from 0.087 to 0.222 (modification by Jenniskens et al. 2018); the agreement between the predictions and this modification is bad only in the case of the models with the high values of $\beta$. No other shower from the IAU MDC list was pointed out up to the unacceptably high $D_{\mathrm{SH}}$-value of 0.407 .

It is, perhaps, worth noting that the $\lambda$-Ursae Majorids were separated from the EDMOND database by Rudawska et al. (2015) in their search for all showers recorded in these data sets by a newly suggested method of separation.

\section{Conclusions}

Assuming that the meteoroids were released from the surface of the long-periodic comet C/1975 T2 (Suzuki-Saigusa-Mori), we modeled its meteoroid stream and this modeling resulted in a prediction of one meteor shower observable in the Earth's atmosphere. This shower was identified with $\lambda$-Ursae Majorids, No. 524 in the IAU MDC list of the established showers. Thus, we confirmed the result of Andreić et al. (2013) and Jenniskens et al. (2016a) who found this shower earlier, also in the videometeor data. Our modeling also confirmed the relationship between the shower and comet C/1975 T2 (Andreić et al. 2013).

The predicted eight-day activity of the shower, from October 21 to 28 , was not confirmed by observations, which imply only a five-day (possibly only two-day) activity, from October 27 to 31 (or October 27 to 28).

The part of orbital corridor of the shower in the terrestrialplanet region, which passes in the vicinity of the Earth's orbit, 
Table 5. Measure of agreement, $\eta$, between the predicted parts of shower related with C/1975 T2 and the modifications of corresponding real shower.

\begin{tabular}{ll|cccccc}
\hline \hline$t_{\mathrm{ev}}$ & $\beta$ & $\mathrm{C}-1$ & $\mathrm{C}-2$ & $\mathrm{~S}-1$ & $\mathrm{~S}-2$ & $\mathrm{E}-1$ & $\mathrm{E}-2$ \\
\hline 10 & $10^{-11}$ & 0.9919 & 1.0000 & 0.6982 & 0.9749 & 0.9369 & 1.0000 \\
10 & 0.0001 & 0.9913 & 1.0000 & 0.6593 & 0.9643 & 0.9056 & 0.9996 \\
10 & 0.001 & 0.9437 & 1.0000 & 0.4773 & 0.8498 & 0.7355 & 0.9956 \\
10 & 0.003 & 0.8817 & 1.0000 & 0.3498 & 0.7499 & 0.6085 & 0.9896 \\
10 & 0.005 & 0.7049 & 1.0000 & 0.0854 & 0.4390 & 0.2841 & 0.9671 \\
20 & $10^{-11}$ & 0.9998 & 1.0000 & 0.9716 & 0.9975 & 0.9784 & 0.9993 \\
20 & 0.0001 & 0.9990 & 1.0000 & 0.9708 & 0.9966 & 0.9791 & 0.9997 \\
20 & 0.001 & 0.9980 & 1.0000 & 0.9361 & 0.9912 & 0.9624 & 0.9996 \\
20 & 0.003 & 0.9807 & 1.0000 & 0.7979 & 0.9387 & 0.8564 & 0.9978 \\
20 & 0.005 & 0.9706 & 1.0000 & 0.6440 & 0.8754 & 0.7274 & 0.9953 \\
20 & 0.007 & 0.8456 & 1.0000 & 0.4430 & 0.7383 & 0.6174 & 1.0000 \\
40 & $10^{-11}$ & 0.9330 & 1.0000 & 0.7607 & 0.9379 & 0.8684 & 0.9938 \\
40 & 0.0001 & 0.9333 & 1.0000 & 0.7493 & 0.9324 & 0.8642 & 0.9930 \\
40 & 0.001 & 0.8949 & 1.0000 & 0.7134 & 0.9088 & 0.8486 & 0.9945 \\
40 & 0.003 & 0.7143 & 1.0000 & 0.4906 & 0.7281 & 0.6567 & 0.9799 \\
40 & 0.005 & 0.5604 & 1.0000 & 0.2825 & 0.5589 & 0.4681 & 0.9626 \\
40 & 0.007 & 0.1723 & 1.0000 & 0.0235 & 0.2689 & 0.1723 & 0.8956 \\
40 & 0.008 & 0.0000 & 1.0000 & 0.0000 & 0.1429 & 0.0000 & 0.9286 \\
80 & $10^{-11}$ & 0.7210 & 0.9815 & 0.4252 & 0.6618 & 0.5055 & 0.9008 \\
80 & 0.0001 & 0.6980 & 0.9834 & 0.4243 & 0.6349 & 0.4886 & 0.8734 \\
80 & 0.001 & 0.6666 & 0.9748 & 0.4530 & 0.6434 & 0.4936 & 0.8905 \\
80 & 0.003 & 0.4278 & 0.9247 & 0.2995 & 0.4333 & 0.3289 & 0.7454 \\
80 & 0.005 & 0.2696 & 0.8513 & 0.1561 & 0.2589 & 0.1788 & 0.5828 \\
80 & 0.007 & 0.0444 & 0.6963 & 0.0160 & 0.0355 & 0.0178 & 0.2842 \\
80 & 0.008 & 0.0000 & 0.1364 & 0.0000 & 0.0000 & 0.0000 & 0.0000 \\
\hline
\end{tabular}

Notes. Each predicted part is characterized with $t_{\mathrm{ev}}$ and $\beta$ (having the same meaning as in Tables 1 and 2). The modifications of real shower were separated from C, S, and E databases (see Tables 3 and 4).

has not changed much during the at least last 80 millennia. This is the reason why $\mathrm{C} / 1975 \mathrm{~T} 2$ produces only a single shower in the Earth's atmosphere. In conclusion, comet C/1975 T2 is the parent body of single, established shower, $\lambda$-Ursae Majorids, No. 524.

Acknowledgements. This article was supported by the realization of the Project ITMS No. 26220120029, based on the supporting operational Research and development program financed from the European Regional Development Fund The work was also supported, in part, by the VEGA - the Slovak Grant Agency for Science, grant No. 2/0037/18, and by the Slovak Research and Development Agency under the contract No. APVV-16-0148.

\section{References}

Abedin, A., Spurný, P., Wiegert, P., et al. 2015, Icarus, 261, 100

Abedin, A., Wiegert, P., Pokorný, P., \& Brown, P. 2017, Icarus, 281, 417

Abedin, A., Wiegert, P., Janches, D., et al. 2018, Icarus, 300, 360

Andreić, Ž., Šegon, D., Korlević, K., et al. 2013, WGN, J. Int. Meteor Organ., 41, 103

Babadzhanov, P. B., Kokhirova, G. I., \& Obrubov, Y. V. 2015a, Sol. Syst. Res., 49, 165

Babadzhanov, P. B., Kokhirova, G. I., \& Khamroev, U. K. 2015b, Adv. Space Res., 55, 1784

Babadzhanov, P. B., Kokhirova, G. I., Williams, I. P., \& Obrubov, Y. V. 2017, A\&A, 598, A94

Chambers, J. E. 1999, MNRAS, 304, 793

Chambers, J. E., \& Murison, M. A. 2000, AJ, 119, 425

Dumitru, B. A., Birlan, M., \& Nedelcu, D. A. 2018, Rom. Astron. J., 28, 167

Everhart, E. 1985, Astrophys. Space Sci. Lib., 115, 185

Gajdoš, S. . 2008, Earth Moon Planets, 102, 117

Giorgini, J. D., Yeomans, D. K., Chamberlin, A. B., et al. 1996, BAAS, 28, 1158

Guennoun, M., Vaubaillon, J., Čapek, D., Koten, P., \& Benkhaldoun, Z. 2019, A\&A, 622, A84
Gural, P. S. 2011, in Proceedings of the International Meteor Conference, 29th IMC, Armagh, Northern Ireland, 2010, 28

Hajdukova, M., Rudawska, R., Kornos, L., \& Toth, J. 2015, Planet. Space Sci., 118,28

Hawkins, G. S. 1963, Smithson. Contrib. Astrophys., 7, 53

Ishiguro, M., Kuroda, D., Hanayama, H., et al. 2015, ApJ, 798, L34

Jenniskens, P., \& Nénon, Q. 2016, Icarus, 266, 371

Jenniskens, P., Gural, P. S., Dynneson, L., et al. 2011, Icarus, 216, 40

Jenniskens, P., Nénon, Q., Gural, P. S., et al. 2016a, Icarus, 266, 355

Jenniskens, P., Nénon, Q., Albers, J., et al. 2016b, Icarus, 266, 331

Jenniskens, P., Nénon, Q., Gural, P. S., et al. 2016c, Icarus, 266, 384

Jenniskens, P., Lyytinen, E., \& Williams, G. V. 2017, CBET, 4403

Jenniskens, P., Baggaley, J., Crumpton, I., et al. 2018, Planet. Space Sci., 154, 21

Jones, P. 2018, eMeteorNews, 3, 38

Jopek, T. J. 2015, Highlights Astron., 16, 474

Jopek, T. J., \& Kaňuchová, Z. 2014, Meteoroids 2013, 353

Jopek, T. J., \& Williams, I. P. 2015, Highlights Astron., 16, 143

Klačka, J. 2014, MNRAS, 443, 213

Kornoš, L., Koukal, J., Piffl, R., \& Tóth, J. 2014a, in Proc. Int. Meteor Conf., Poznan, Poland, 22-25 August 2013, eds. M. Gyssens, P. Roggemans, \& P. Zoladek (Hove, Belgium: International Meteor Organization), 23

Kornoš, L., Matlovič, P., Rudawska, R., et al. 2014b, Meteoroids, 2013, 225

Kornoš, L., Tóth, J., Porubčan, V., et al. 2015, Planet. Space Sci., 118, 48

Koten, P., \& Vaubaillon, J. 2015, Eur. Planet. Sci. Congress, 10, EPSC2015

Koukal, J. 2018, eMeteorNews, 3, 1

Molau, S., Crivello, S., Goncalves, R., et al. 2018a, WGN, J. Int. Meteor Organ., 46, 142

Molau, S., Crivello, S., Goncalves, R., et al. 2018b, WGN, J. Int. Meteor Organ., 46, 166

Molau, S., Crivello, S., Goncalves, R., et al. 2018c, WGN, J. Int. Meteor Organ., 46, 205

Muirden, J., Andersson, K. G., Bortle, J., Guth, V., \& Hasegawa, I. 1975, IAU Circ., 2858

Neslušan L. 1999, A\&A, 351, 752

Neslušan, L., Svoreň, J., \& Porubčan, V. 1995, Earth Moon Planets, 68, 427

Neslušan, L., Svoreň, J., \& Porubčan, V. 2013, Earth Moon Planets, 110, 41

Neslušan, L., Porubčan, V., \& Svoreň, J. 2014, Earth Moon Planets, 111, 105

Olech, A., Żoładek, P., Wiśniewski, M., et al. 2016, MNRAS, 461, 674

Porubčan, V., Svoreň, J., Neslušan, L., \& Schunová, E. 2011, in Meteoroids: The Smallest Solar System Bodies, eds. W. J. Cooke, D. E. Moser, B. F. Hardin, \& D. Janches (Hanover: NASA), 338

Rudawska, R., \& Vaubaillon, J. 2015, Planet. Space Sci., 118, 25

Rudawska, R., Matlovic, P., Toth, J., Kornos, L., \& Hajdukova, M. 2015, European Planetary Science Congress, 10, EPSC2015

Rudawska, R., Vaubaillon, J., Tóth, J., \& Raetz, S. 2016, AAS/Division Planet. Sci. Meeting Abstracts, 48, 218.01

Ryabova, G. O. 2016, MNRAS, 456, 78

Ryabova, G., \& Rendtel, J. 2018a, European Planetary Science Congress, 12, EPSC2018

Ryabova, G. O., \& Rendtel, J. 2018b, MNRAS, 475, L77

Ryabova, G. O., Avdyushev, V. A., \& Williams, I. P. 2019, MNRAS, 485, 3378

Šegon, D., Vaubaillon, J., Gural, P. S., et al. 2017, A\&A, 598, A15

Sekanina, Z., \& Southworth, R. B. 1975, Physical and dynamical studies of meteors. Meteor-fragmentation and stream-distribution studies, Tech. rep.

Sergienko, M. V., Sokolova, M. G., Andreev, A. O., \& Nefedyev, Y. A. 2018a, LPI Contrib., 2067, 6162

Sergienko, M. V., Sokolova, M. G., Andreev, A. O., \& Nefedyev, Y. A. 2018b, LPI Contrib., 2067, 6165

Shiba, Y., Shimoda, C., Maeda, K., et al. 2018, WGN, J. Int. Meteor Organ., 46, 79

SonotaCo. 2009, WGN, J. Int. Meteor Organ., 37, 55

SonotaCo. 2016, WGN, J. Int. Meteor Organ., 44, 42

Southworth, R. B., \& Hawkins, G. S. 1963, Smithson. Contrib. Astrophys., 7, 261

Sugar, G., Moorhead, A., Brown, P., \& Cooke, W. 2017, Meteorit. Planet. Sci., 52,1048

Tomko, D., \& Neslušan, L. 2012, Earth Moon Planets, 108, 123

Tomko, D., \& Neslušan, L. 2019, A\&A, 623, A13

Toth, J., Kornos, L., Matlovic, P., et al. 2018, European Planetary Science Congress, 12, EPSC2018

Vaubaillon, J. 2017, Planet. Space Sci., 143, 78

Vida, D., Merlak, A., \& Šegon, D. 2018a, eMeteorNews, 3, 276

Vida, D., Merlak, A., \& Segon, D. 2018b, eMeteorNews, 3, 298

Wiegert, P., Clark, D., Campbell-Brown, M., \& Brown, P. 2017, CBET, 4415

Wisniewski, M., Zoladek, P., Olech, A., \& Jonderko, W. 2018, WGN, J. Int. Meteor Organ., 46, 30

Ye, Q.-Z. 2018, Planet. Space Sci., 164, 7 